\title{
The Ecological Dynamics of 1v1 Sub-Phases in Association Football
}

\author{
R. Duarte ${ }^{1,2, *}$, D. Araújo ${ }^{2}$, V. Gazimba ${ }^{1}$, O. Fernandes ${ }^{1}$, H. Folgado $^{1}$, J. Marmeleira ${ }^{1}$ and K. Davids ${ }^{3}$ \\ ${ }^{I}$ Department of Sport and Health, University of Évora, Portugal \\ ${ }^{2}$ Faculty of Human Kinetics, Technical University of Lisbon, Portugal \\ ${ }^{3}$ School of Human Movement Studies, Queensland University of Technology, Australia
}

\begin{abstract}
The purpose of this study was to analyze the informational constraints that influence the dynamics of $1 \mathrm{v} 1$ situations in football. Specifically, we analyzed the contribution of interpersonal distance and relative velocity to explain phase transitions that occurred between stable and unstable coordinative states in 1v1 in football. Participants were six, U13 level football players (Mean age: $11.8 \pm 0.4 \mathrm{yrs}$ ), who performed 5 trials of a representative task at four different start distances (1, 1.5, 2 and 2.5 meters) between the defender and the ball ( 5 repetitions x 6 players x 4 distances $=1201 \mathrm{v} 1$ trials). Players' motion was captured by digital video camera. For image treatment and to extract data on player movement coordinates, we used the TACTO 8.0 software at $25 \mathrm{~Hz}$. Following, we constructed plots with the time-series of these two control parameters candidates associated with the time-series of players' distance to the end line. Graphical inspection showed in all trials a clear tendency for greater relative velocity and lower interpersonal distance values during phase transitions between coordinative states. To confirm this interpretation, we computed a time-series analysis of Z-scores of relative velocity and interpersonal distance data, and a time-series analysis of $Z$-score differences between these variables. Mean data showed that maximum peaks in Z-score differences were related to all phase transitions.
\end{abstract}

Keywords: Ecological dynamics, Interpersonal distance, Relative velocity, Informational constraints.

\section{INTRODUCTION}

Association football constitutes an open complex system characterized by adaptive behaviour as result of dynamic interactions of the players [1]. Interpersonal interactions at a microscopic level of organization (e.g., between attackers and defenders in $1 \mathrm{v} 1$ sub-phases) can prompt nonlinear transitions in macroscopic patterns of behaviour (e.g., patterns of play in games) [2]. Despite the enormous variability and complexity of a football match, theoretical and experimental evidence suggests the existence of dynamic patterns of interpersonal coordination between players, at various levels of analysis [3]. These pattern-forming dynamics emerge in the complex interpersonal interactions of performers regulated through their direct perceptual processes [4,5]. The 1v1 sub-phase near a target/goal area is the most studied sub-system in team sports from this perspective. For example, in basketball, critical values of interpersonal distance were identified as a potential control parameter that pushes the system to phase transitions [6]. In rugby, interpersonal distance has also been identified as a candidate control parameter [7]. However, that research showed that for interpersonal distances values of less than $4 \mathrm{~m}$, relative velocity assumed crucial importance as the control parameter responsible for the phase transitions in the dyadic systems [7]. In football, the difference between the distance of the attacker and the defender to a target zone (for example, the goal or

*Address correspondence to this author at the Department of Sport and Health, University of Évora, Portugal; Tel: 00351214196777;

Fax: 00351214144712; E-mail: rduarte@fmh.utl.pt the free space behind the defender) can be defined as a potential order parameter $[3,8]$, identifying two qualitative states: (1) when the defender is nearer the target zone than the attacker, and (2) when the attacker passes the defender and approaches the target zone. Based on the ecological dynamics approach [5], the purpose of this study was to investigate the informational constraints that influence the dynamics of 1v1 sub-phases in football. Specifically, our aim was to analyze interpersonal distance and relative velocity as control parameter candidates that influence the phase transition between the two organizational states in $1 \mathrm{v} 1 \mathrm{sub}-$ phases of association football.

\section{METHODS}

Participants were six, U13 experienced football players (Mean age: $11.8 \pm 0.4 \mathrm{yrs}$; training experience: $3.6 \pm 1.1 \mathrm{yrs}$ ). The task consisted of a representative situation in which the attacker tried to destabilize a dyad formed with a defender to explore the free space behind him and create shooting opportunities. In addition, the defender tried to maintain dyadic system stability by preventing the attacker from shooting (see Fig. 1).

Players performed five $1 \mathrm{v} 1$ trials each at four different start distances $(1,1.5,2$ and $2.5 \mathrm{~m})$ between the defender and the ball $(5$ repetitions x 6 players $\times 4$ distances $=1201 \mathrm{v} 1$ trials). Based on previous criteria, we excluded 31 null trials and accepted 89 for further analysis. To simulate performance constraints, we created a $1 \mathrm{v} 1$ competition between players. Players' motion was captured by digital video camera. For image treatment and to extract data on player movement coordinates, we used the TACTO 8.0 software 
[9], digitizing action at $25 \mathrm{~Hz}$. The procedure consisted of following with the mouse the earlier tested working point in the middle of the feet. After 7 days of training, the reliability of digitizing action was $\mathrm{r}=0.99$ to $\mathrm{x}$, and $\mathrm{r}=0.98$ to $\mathrm{y}$ coordinates. After calibration with real measures of six control points, the $\mathrm{x}$ and $\mathrm{y}$ virtual coordinates of the players were extracted. To transform the virtual into real coordinates we used the Direct Linear Transformation Parameters (PDLT) [10]. Subsequently, position and velocity data of all players in all trials were computed and analyzed with MATLAB software.

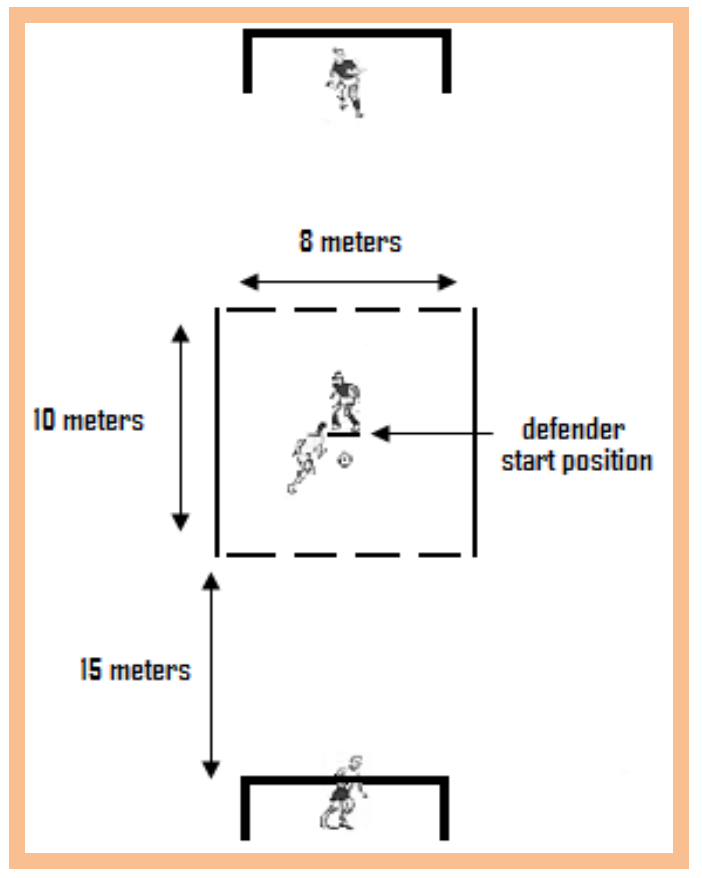

\section{RESULTS}

Results showed no statistical differences for all outcome measures analyzed to discriminate differences between the various initial start distances (as in previous research on basketball and rugby union $[6,7]$ we studied percentage of time spent in symmetry, anti-symmetry and undifferentiated state; time and distance to the target zone of first and last phase transitions; and the number of zero crossings).

To analyze the contribution of interpersonal distance and relative velocity as variables to explain phase transitions in $1 \mathrm{v} 1$ sub-phases of football, we constructed plots of the timeseries of these two candidate control parameters related to the time-series of players' distance to the target zone. Graphical inspection of the data showed that in all trials there was a clear tendency for higher relative velocity and lower interpersonal distance values during phase transitions between the two coordinative states (see Fig. 2).

To confirm this interpretation, we computed a time-series analysis of Z-scores of relative velocity and interpersonal distance data, and a time-series analysis of Z-score differences between these variables (see Fig. 3). Mean data showed that maximum peaks in Z-score differences were related to all phase transitions.

\section{DISCUSSION}

Analysis of the moment of phase transitions showed that this instant was related to lower values of interpersonal distance and higher values of relative velocity. When these control parameters were expressed as a single variable (see Zscore difference in Fig. 3), maximum peaks were related to the exact moment of phase transition. The findings of the present study suggest that dyadic system behaviours emerge in an environmental exploratory process $[4,6]$ that cannot be explained exclusively by only one control parameter. These results are similar to the study of $1 \mathrm{v} 1$ sub-phases in rugby union [7], in which it was shown that for interpersonal dis-

Fig. (1). Experimental task schematic representation.

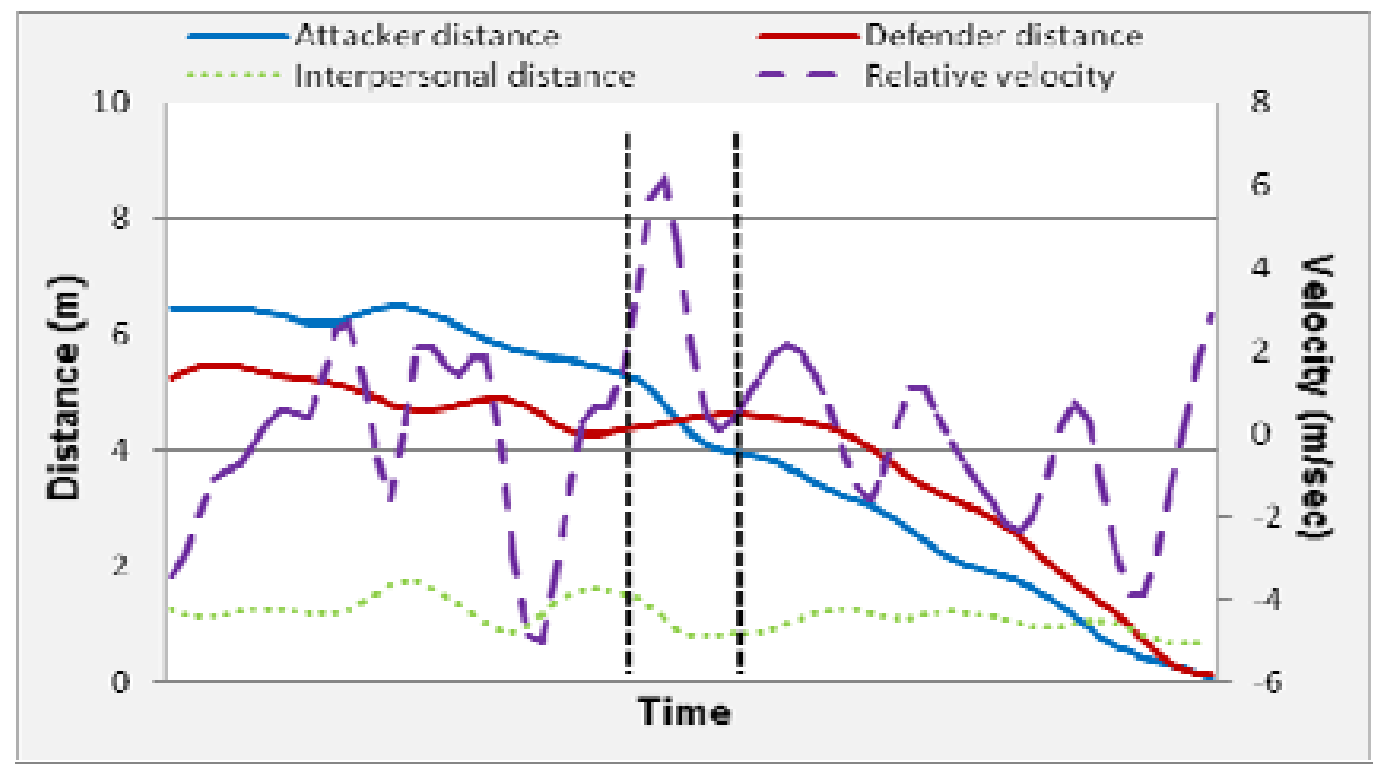

Fig. (2). Example of the identified tendency in one random selected trial. 


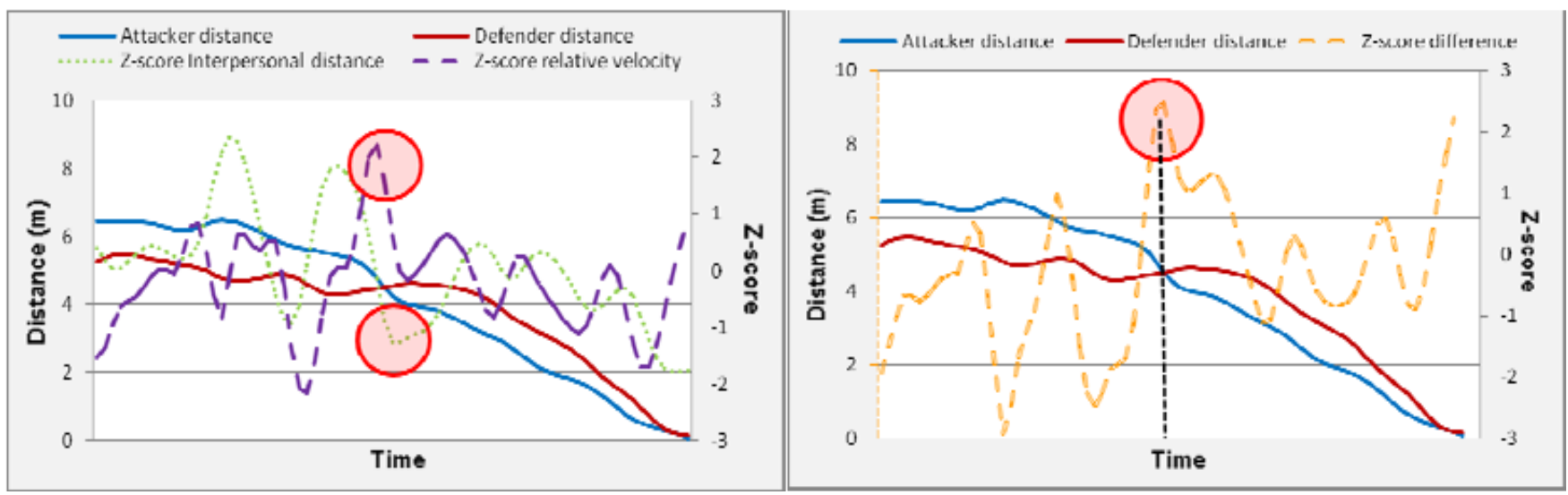

Fig. (3). Time-series of Z-scores in the same trial. On the left, Z-scores of relative velocity and interpersonal distance showed higher and lower values associated to phase transition. On the right, the difference between Z-scores of relative velocity and interpersonal distance (Zscore difference) showed a maximum peak in the exact moment of the phase transition.

tance values of less than $4 \mathrm{~m}$, relative velocity was the control parameter responsible for the phase transitions. In this study of association football, to represent the specific task constraints of $1 \mathrm{v} 1$ sub-phases, the different initial start distances were less than $4 \mathrm{~m}$ (between 1 and 2.5 meters). This fact can attributed to the importance assumed for relative velocity in destabilizing the attacker-defender dyad. However, this control parameter was strongly associated with lower values of interpersonal distances in all trials. Summarizing, at critical values of interpersonal distance in football, attackers can destabilize dyads by altering the difference between his/her velocity and the defender's velocity. These results have several implications for training task design in football. Attackers should be encouraged to explore the exact moment at which the affordance to drive past a defender emerges [3-5]. These affordances emerge at lower values of interpersonal distance, particularly when the difference between the relative velocities of the players is higher. On the other hand, defenders should be encouraged to maintain appropriate interpersonal distance with attackers to maintain stability of the 1v1 sub-system.

Moreover, we observed that the same physical variables constrained the dynamics of performer-environment interactions despite variations in initial start distances between players. This observation shows high levels of consistency in the players using the same informational constraints to guide their actions in $1 \mathrm{v} 1 \mathrm{sub}$-phases of football.

\section{REFERENCES}

[1] Gréhaigne JF, Bouthier D, David B. Dynamic-system analysis of opponent relationships in collective actions in soccer. J Sports Sci 1997; 15: 137-49.

[2] Davids K, Araújo D, Shuttleworth R. Applications of dynamical systems theory to football. In: Reilly T, Cabri J, Araújo D, Eds. science and football V. Oxon: Routledge, Taylor \& Francis 2005: pp. $537-50$

[3] Schmidt RC, O’Brien B, Sysko R. Self-organization of between person cooperative tasks and possible applications to sport. Int $\mathbf{J}$ Sport Psychol 1999; 30: 558-79.

[4] Fajen BR, Riley MA, Turvey MT. Information, affordances, and the control of action in sport. Int J Sport Psychol 2008; 40: 79-107.

[5] Araújo D, Davids K, Hristovski R. The ecological dynamics of decision making in sport. Psych Sport Exerc 2006; 7: 653-76.

[6] Araújo D, Davids K, Bennett SJ, Button C, Chapman G. Emergence of sport skills under constraints. In: Williams AM, Hodges NJ, Eds. Skill acquisition in sport: research, theory and practice. London: Routledge, Taylor \& Francis 2004; pp. 409-33.

[7] Passos P, Araújo D, Davids K, Gouveia L, Milho J, Serpa S. Information-governing dynamics of attacker-defender interactions in youth rugby union. J Sports Sci 2008; 26: 1421-9.

[8] McGarry T, Anderson D, Wallace S, Hughes M, Franks I. Sport competition as a dynamical self-organizing system. J Sports Sci 2002; 15: 171-81.

[9] Fernandes O, Folgado H, Duarte R, Malta P. Validation of the tool for applied and contextual Time-series observation. Int J Sport Psychol 2010 (in press).

[10] Abdel-Aziz YI, Karara HM. Direct linear transformation from comparator coordinates into object space coordinates in close-range photogrammetry. In: Proceedings of the Symposium on CloseRange Photogrammetry; Falls Church, VA, United States. American Society of Photogrammetry 1971; pp. 1-18.

(C) Duarte et al.; Licensee Bentham Open.

This is an open access article licensed under the terms of the Creative Commons Attribution Non-Commercial License (http://creativecommons.org/licenses/by-nc/3.0/) which permits unrestricted, non-commercial use, distribution and reproduction in any medium, provided the work is properly cited. 